\title{
Stochastic dynamics of genetic broadcasting networks
}

\author{
Davit A. Potoyan and Peter G. Wolynes \\ Department of Chemistry and Center for Theoretical Biological Physics, Rice University, Houston, Texas 77005, USA
}

(Received 1 May 2017; revised manuscript received 10 July 2017; published 3 November 2017)

\begin{abstract}
The complex genetic programs of eukaryotic cells are often regulated by key transcription factors occupying or clearing out of a large number of genomic locations. Orchestrating the residence times of these factors is therefore important for the well organized functioning of a large network. The classic models of genetic switches sidestep this timing issue by assuming the binding of transcription factors to be governed entirely by thermodynamic protein-DNA affinities. Here we show that relying on passive thermodynamics and random release times can lead to a "time-scale crisis" for master genes that broadcast their signals to a large number of binding sites. We demonstrate that this time-scale crisis for clearance in a large broadcasting network can be resolved by actively regulating residence times through molecular stripping. We illustrate these ideas by studying a model of the stochastic dynamics of the genetic network of the central eukaryotic master regulator $\mathrm{NF} \kappa \mathrm{B}$ which broadcasts its signals to many downstream genes that regulate immune response, apoptosis, etc.
\end{abstract}

DOI: 10.1103/PhysRevE.96.052305

\section{INTRODUCTION}

During development gene regulatory programs translate the information in the genome into phenotypes and after development these programs then micromanage many functions within cells in order to control their survival in a changing environment. At the molecular level, regulation involves an intricate web of interactions where the protein products of one set of genes bind to other genes and their products so as to modulate the production of biomolecules. The simplest element of a gene regulatory network is a genetic switch which can be thought of as a cellular control unit that can turn on or off in response to external stimuli or signals from other genes [1]. The earliest models of genetic switches were formulated using purely thermodynamic models $[2,3]$. These models assumed that gene states are rapidly equilibrated

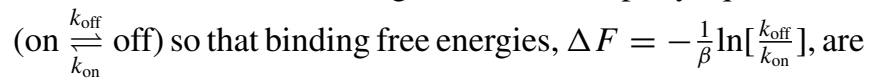
the sole quantities controlling the systems level behavior of the network. These models, first introduced in studies on bacteria [4,5], are often used to explain gene expression patterns of higher organisms also in terms of protein-DNA interaction affinities. Thermodynamic models indeed have been fruitful in interpreting Chip-seq and binding microarray data and thereby have served as a conceptual link between molecular structure and gene expression [3,6]. In vivo, however, eukaryotic networks are generally far from equilibrium, and the switching between states of a gene involves many elaborate kinetically controlled steps such as conformational changes of chromatin, assembly of various protein cofactors into larger transcription complexes, RNA polymerase attachment, etc. [7-9]. The apparently simple concept of a gene switching in response to equilibrium binding is therefore a high level idealization which may not be universally applicable.

Under time varying nonequilibrium conditions the in vivo activity of genes will be dictated not only by equilibrium binding but also by the residence time of transcription factors once they are bound to the DNA. Recent single cell and single molecule studies [10-12] show there are significant departures from the predictions of conventional thermodynamic models. Single molecule chase assay experiments which directly measure the dissociation rates of transcription factors are physically inconsistent with the naive equilibrium models for the genetic switch [11]. A number of possibilities have been proposed to account for the departures from thermodynamic models $[7,11]$. Some have argued that energy consuming kinetic proofreading schemes could be employed by eukaryotic cells for attaining greater sensitivity [13] and specificity [14] of transcription factor binding relative to binding in equilibrium.

One intriguing mechanism that has been largely overlooked is that of an active regulation of the unbinding step through induced molecular stripping processes which remove transcription factors from their genomic binding sites $[15,16]$. While some transcription factors may be spontaneously released from bound complexes the in vitro kinetic studies of the important transcription factor, $\mathrm{NF} \kappa \mathrm{B}$, interacting with DNA and its inhibitor $\mathrm{I} \kappa \mathrm{B}$ have suggested that the regulation of $\mathrm{NF} \kappa \mathrm{B}$ in cells is likely to be kinetically controlled directly by the inhibitor [15,17]. Molecular dynamics simulations have provided a detailed molecular level picture [16] of how $\mathrm{I} \kappa \mathrm{B} \alpha$ actively strips the transcription factor off of DNA more rapidly than passive dissociation could. In addition, in recent years single molecule level experiments have uncovered other cases of active regulation where protein-DNA exchange involves the formation of ternary complexes and consequent concentration dependent dissociation of proteins from DNA [10]. Facilitated dissociation has been observed in systems as diverse as the nonspecifically bound architectural proteins $[18,19]$, metal sensing transcriptional regulators [12,20], RNA polymerase [21] and even in ribosomal subunit switching [22].

Unlike the bacterial switches studied in the golden age of the molecular biology of bacteria, $\mathrm{NF} \kappa \mathrm{B}$ does not act as a simple switch turning on a single metabolic pathway but is involved in a very wide range of regulatory activities in eukaryotic cells [23]. To carry out these activities turning on the $\mathrm{NF} \kappa \mathrm{B}$ switch broadcasts a signal to many downstream genes. As we shall discuss in the paper, this broadcasting responsibility of $\mathrm{NF} \kappa \mathrm{B}$ creates a severe problem of timing if only passive dissociation of $\mathrm{NF} \kappa \mathrm{B}$ from its targets is possible. The broadcasting network models with numerous DNA binding sites that do not account for this active regulation of dissociation times encounter a "time-scale crisis." We argue this crisis is a general phenomenon in genetic broadcasting 
systems lacking active regulation of dissociation. Owing to the large genomes and complex life styles of eukaryotes, $\mathrm{NF} \kappa \mathrm{B}$ like many other master switches has a huge number of target sites that initiate downstream functions [24,25]. There are also myriads of apparently nonfunctional sites where $\mathrm{NF} \kappa \mathrm{B}$ binds [26]. The $\mathrm{NF} \kappa \mathrm{B}$ activity coordinates a symphony of genes in response to complex environmental stimuli. Once the external environment returns to normal, however, not only is there no longer any need for further expression of these $\mathrm{NF} \kappa \mathrm{B}$ target genes but if they are not promptly turned off deleterious actions may result. In contrast, as we shall see, by employing molecular stripping, the concentration of free and transcriptionally active $\mathrm{NF} \kappa \mathrm{B}$ will be promptly titrated back to zero by its inhibitor $\mathrm{I} \kappa \mathrm{B}$ once the stimulus is turned off. In many previous models of the $\mathrm{NF} \kappa \mathrm{B} / \mathrm{I} \kappa \mathrm{B}$-DNA circuitry $\mathrm{I} \kappa \mathrm{B}$ was thought to simply wait to encounter $\mathrm{NF} \kappa \mathrm{B}$ molecules that had become unbound passively from the DNA in order to finally remove $\mathrm{NF} \kappa \mathrm{B}$ from the nucleus. We shall see that waiting for the $\mathrm{NF} \kappa \mathrm{B}$ to unbind completely from tens of thousands of sites in order to become available for the freely diffusing $\mathrm{I} \kappa \mathrm{B}$ simply takes too much time. Facing a changing environment, time becomes essential to the organism. The $\mathrm{I} \kappa \mathrm{B}$ induced direct stripping of $\mathrm{NF} \kappa \mathrm{B}$ from its genetic sites prevents this time-scale crisis. In our view, regulating residence times is a necessity for genetic circuitry that broadcasts signals to multiple targets.

\section{THE NF $\kappa$ B/I $\kappa$ B-DNA BROADCASTING NETWORK}

The transcription factors of the $\mathrm{NF} \kappa \mathrm{B}$ family are present in large quantities in eukaryotic cells $\left(\sim 10^{5}\right.$ copies per cell). They activate $\sim 5 \times 10^{2}$ different genes [27,28] in response to external stimuli. Due to its wide ranging influence over so many signaling activities $\mathrm{NF} \kappa \mathrm{B}$ can be regarded as a typical master regulator switch which "broadcasts" signals to many target genes. Here we employ a stochastic model of the $\mathrm{NF} \kappa \mathrm{B}$ broadcasting network which includes the core inhibitory feedback loop of $\mathrm{I} \kappa \mathrm{B}$ along with the many DNA targets and decoy sites to which $\mathrm{NF} \kappa \mathrm{B}$ binds including, most importantly, the promoter for $\mathrm{I} \kappa \mathrm{B}$ itself (Fig. 1 and Table I). The $\mathrm{I} \kappa \mathrm{B}$ feedback loop is activated under external stimulation when $\mathrm{NF} \kappa \mathrm{B}-\mathrm{I} \kappa \mathrm{B}$ is marked for degradation of $\mathrm{I} \kappa \mathrm{B}$. Once freed from $\mathrm{I} \kappa \mathrm{B}, \mathrm{NF} \kappa \mathrm{B}$ goes inside the nucleus and binds to a myriad of DNA sites, including the promoter of $\mathrm{I} \kappa \mathrm{B}$ itself. Binding to the promoter site initiates the synthesis of $\mathrm{I} \kappa \mathrm{B}$ which then repeats the cycle as long as the external stimulation is present. Due to long delayed negative feedback by $\mathrm{I} \kappa \mathrm{B}$, the network shows undamped oscillations of $\mathrm{NF} \kappa \mathrm{B} / \mathrm{I} \kappa \mathrm{B}$ at the single cell level under constant stimulation [29-31]. The removal of stimulation is followed by clearance of $\mathrm{NF} \kappa \mathrm{B}$ from the nucleus and return to the steady state where all of the $\mathrm{NF} \kappa \mathrm{B}$ is retained in cytoplasm by the $\mathrm{I} \kappa \mathrm{B}$ until the encounter of a new signal. The model of $\mathrm{NF} \kappa \mathrm{B}$ considered here is doubtless a drastic simplification of biological complexity present in eukaryotic cells. Nevertheless we show that a tight negative loop and the presence of a large number of binding sites are sufficient to illustrate the existence of a time-scale crisis and its resolution via a kinetic control of the transcription factor's residence times or in the case of $\mathrm{NF} \kappa \mathrm{B}$ the molecular stripping.
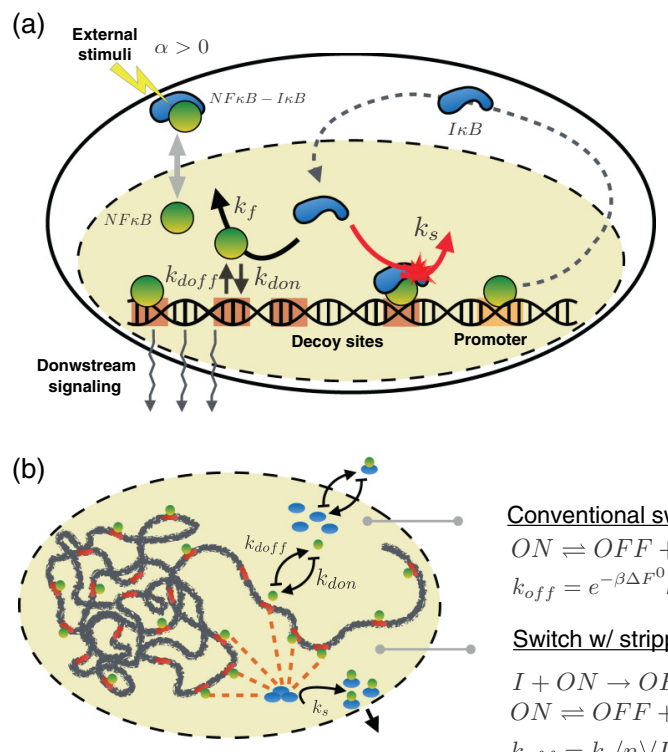

$$
\begin{aligned}
& \text { Conventional switch } \\
& O N \rightleftharpoons O F F+T F \\
& k_{o f f}=e^{-\beta \Delta F^{0}} k_{o n}(n) \\
& \text { Switch w/ stripping } \\
& I+O N \rightarrow O F F \\
& O N \rightleftharpoons O F F+T F \\
& k_{o f f}=k_{s}\langle n\rangle\langle I\rangle+e^{-\beta \Delta F^{0}} k_{o n}(n)
\end{aligned}
$$

FIG. 1. (a) Schematic picture of the broadcasting network of master regulator $\mathrm{NF} \kappa \mathrm{B}$. Under external stimulation $\mathrm{NF} \kappa \mathrm{B}-\mathrm{I} \kappa \mathrm{B}$ is marked for degradation of $\mathrm{I} \kappa \mathrm{B}$ with the rate $\alpha$. Once freed, $\mathrm{NF} \kappa \mathrm{B}$ goes inside the nucleus and binds to a myriad of DNA sites, including functional targets for downstream signaling, nonfunctional decoys, and the promoter for $\mathrm{I} \kappa \mathrm{B}$ itself. Binding to the promoter site initiates the synthesis of $\mathrm{I} \kappa \mathrm{B}$. The negative feedback loop of $\mathrm{I} \kappa \mathrm{B}$ is shown with the dashed line with its inhibition of $\mathrm{NF} \kappa \mathrm{B}$ taking place either via direct binding to dissociated $\mathrm{NF} \kappa \mathrm{B}$ (solid black arrow) or via molecular stripping. (b) Shown are the mechanisms of (1) the broadcasting network with no stripping, where the DNA unbinding rate takes place via passive dissociation, and (2) the switch with stripping, where switching is being controlled kinetically via broadcasting signals of $\mathrm{I} \kappa \mathrm{B}$ stripping $\mathrm{NF} \kappa \mathrm{B}$ off of DNA sites directly in addition to a passive dissociation step.

The target genes which are activated by the binding of $\mathrm{NF} \kappa \mathrm{B}$ to initiate signaling activities downstream are accompanied by a large majority of genomic sites that do not code for proteins and are likely decoys serving no known functions. An order of magnitude estimate for the effective number of decoys comes from genome-wide Chip-seq assays of binding [26] which have singled out more than $2 \times 10^{4}$ different DNA sites that bind $\mathrm{NF}_{\kappa} \mathrm{B}$. Our usage of the word "decoy" in the discussion below warrants some further explanation. Aside from the promoter of $\mathrm{I} \kappa \mathrm{B}$, all the other $\mathrm{NF} \kappa \mathrm{B}$ binding sites will be treated in the same way as being effectively homogeneous binding sites obtained by dividing the accessible part of the genome into finite nonoverlapping stretches of DNA, each of which binds a single $\mathrm{NF} \kappa \mathrm{B}$ molecule. Using the effective decoy concept allows us to average out sequence dependent effects. In the present paper the effective binding sites are nearly identical entities with comparable residence times. The heterogeneity of binding and release properties of different sites is certainly an interesting issue (especially from the bioinformatic perspective) to which we plan to return in a future publication. The model network we study contains $D=2 \times 10^{4}$ such effective decoys. The total number of $\mathrm{NF} \kappa \mathrm{B}$ molecules is $N_{\mathrm{NF} \kappa \mathrm{B}}^{\text {tot }}=10^{5}$. Both the number of decoys and the total number of $\mathrm{NF} \kappa \mathrm{B}$ molecules are essentially constant 
TABLE I. Reactions and rate coefficients for the $\mathrm{NF} \kappa \mathrm{B} / \mathrm{I} \kappa \mathrm{B}-\mathrm{DNA}$ broadcasting network. The individual based kinetic Monte Carlo algorithm [33] was used to simulate stochastic dynamics of the network. $G^{\text {on }}$ and $G^{\text {off }}$ denote $\mathrm{NF} \kappa \mathrm{B}$ bound and free states of the $\mathrm{I} \kappa \mathrm{B}$ promoter. $D^{\text {on }}$ and $D^{\text {off }}$ denote $\mathrm{NF} \kappa \mathrm{B}$ bound and free states of decoys. The network has one $\mathrm{I} \kappa \mathrm{B}$ promoter and $2 \times 10^{4}$ effective decoy sites. Irreversible reactions are indicated by the $\Rightarrow$ arrow. The kinetic parameters that are varied in our survey of system behaviors are highlighted in color, and the rest are adopted from kinetic measurements by Hoffmann et al. [32]. Reactions that take place in cytoplasm or after transferring into cytoplasm from the nucleus are indicated by (cyt) and (nuc), and the rest of the process is taken to occur in the nucleus.

\begin{tabular}{lcc}
\hline \hline Reactions & Rate Coeff. & Values \\
\hline $\mathrm{I} \kappa \mathrm{B}-\mathrm{NF} \kappa \mathrm{B} \Rightarrow \mathrm{NF} \kappa \mathrm{B}$ & $\alpha$ & 0 or $0.25 \mathrm{~min}^{-1}$ \\
$D^{\text {off }}+\mathrm{NF} \kappa \mathrm{B} \rightarrow D^{\text {on }}$ & $k_{\text {don }}$ & $10 \mu \mathrm{M}^{-1} \mathrm{~min}^{-1}$ \\
$D^{\text {on }} \rightarrow D^{\text {off }}+\mathrm{NF} \kappa \mathrm{B}$ & $k_{\text {doff }}$ & $0.01-10 \min ^{-1}$ \\
$\mathrm{I} \kappa \mathrm{B}+D^{\text {on }} \Rightarrow \mathrm{I} \kappa \mathrm{B}-\mathrm{NF} \kappa \mathrm{B}+D^{\text {off }}$ & $k_{s}$ & $0-10 \mu \mathrm{M}^{-1} \mathrm{~min}^{-1}$ \\
$\mathrm{I} \kappa \mathrm{B}+G^{\text {on }} \Rightarrow \mathrm{I} \kappa \mathrm{B}-\mathrm{NF} \kappa \mathrm{B}+G^{\text {off }}$ & $k_{s}$ & $0-10 \mu \mathrm{M}^{-1} \min ^{-1}$ \\
$G^{\text {off }}+\mathrm{NF} \kappa \mathrm{B} \rightarrow G^{\text {on }}$ & $k_{\text {on }}$ & $10 \mu \mathrm{M}^{-1} \min ^{-1}$ \\
$G^{\text {on }} \rightarrow G^{\text {off }}+\mathrm{NF} \kappa \mathrm{B}$ & $k_{\text {off }}$ & $0.1 \min ^{-1}$ \\
$G^{\text {on }} \Rightarrow G^{\text {on }}+m \mathrm{RNA}(\mathrm{cyt})$ & $k_{t}$ & $1.0 \mu \mathrm{Mmin}^{-1}$ \\
$m \mathrm{RNA} \Rightarrow m \mathrm{RNA}+I_{c}(\mathrm{cyt})$ & $k_{t l}$ & $0.23 \mathrm{~min}^{-1}$ \\
$m \mathrm{RNA} \Rightarrow \varnothing(\mathrm{cyt})$ & $\gamma_{m}$ & $0.015 \mathrm{~min}^{-1}$ \\
$\mathrm{I} \kappa \mathrm{B}(\mathrm{cyt}) \rightarrow \mathrm{I} \kappa \mathrm{B}(\mathrm{nuc})$ & $k_{I \text { in }}$ & $0.015 \mathrm{~min}^{-1}$ \\
$\mathrm{I} \kappa \mathrm{B}(\mathrm{nuc}) \rightarrow \mathrm{I} \kappa \mathrm{B}(\mathrm{cyt})$ & $k_{\text {out }}$ & $0.012 \mathrm{~min}^{-1}$ \\
$\mathrm{NF} \kappa \mathrm{B}(\mathrm{cyt}) \Rightarrow \mathrm{NF} \kappa \mathrm{B}(\mathrm{nuc})$ & $5.4 \mathrm{~min}^{-1}$ \\
$\mathrm{NF} \kappa \mathrm{B}-I \kappa \mathrm{B}(\mathrm{nuc}) \Rightarrow \mathrm{NF} \kappa \mathrm{B}-\mathrm{I} \kappa \mathrm{B}(\mathrm{cyt})$ & $k_{\text {out }}$ & $0.83 \mathrm{~min}^{-1}$ \\
$\mathrm{NF} \kappa \mathrm{B}+\mathrm{I} \kappa \mathrm{B}(\mathrm{cyt}) \rightarrow \mathrm{NF} \kappa \mathrm{B}-\mathrm{I} \kappa \mathrm{B}(\mathrm{cyt})$ & $k_{f}$ & $30.0 \mu \mathrm{M}^{-1} \mathrm{~min}^{-1}$ \\
$\mathrm{NF} \kappa \mathrm{B}-\mathrm{I} \kappa \mathrm{B}(\mathrm{cyt}) \rightarrow N F \kappa+I \kappa \mathrm{B}(\mathrm{cyt})$ & $k_{b}$ & $0.03 \min ^{-1}$ \\
$\mathrm{NF} \kappa \mathrm{B}+I \kappa \mathrm{B} \rightarrow \mathrm{NF} \kappa \mathrm{B}-\mathrm{I} \kappa \mathrm{B}$ & $k_{f n}$ & $30.0 \mu \mathrm{M}^{-1} \mathrm{~min}^{-1}$ \\
$\mathrm{NF} \kappa \mathrm{B}-\mathrm{I} \kappa \mathrm{B} \rightarrow \mathrm{NF} \kappa \mathrm{B}+I \kappa \mathrm{B}$ & $k_{b n}$ & $0.03 \min ^{-1}$ \\
\hline \hline
\end{tabular}

owing to the long cellular lifetime of $\mathrm{NF} \kappa \mathrm{B}$. It should be noted that these effective decoys have larger capture rates and residence times for sliding along the DNA compared to the short consensus sequences of NF $\kappa \mathrm{B}$ where binding has been studied in vitro. The parameters and reaction rates used in the model are shown in Table I. The values for the rates of binding and unbinding steps are adopted from in vitro DNA binding experiments [15,17] and genome wide microarray data [25]. The rest of the rates come from bulk kinetic experiments [32]. The binding on rates are mostly diffusion limited and are set to $k_{\mathrm{on}}=k_{\mathrm{don}}=10 \mu \mathrm{M} \mathrm{min}^{-1}$. The off rates, on the other hand, show greater variation [25] and generally fall in the range between $10^{-2}$ and $10 \mathrm{~min}^{-1}$. To understand the generality of the issue we vary the effective decoy off rates $k_{\text {doff }}$ in this range in order to obtain a complete survey of dynamic regimes that may be exhibited not only by this system but also by other analogous broadcasting networks. The off rate for the single $\mathrm{I} \kappa \mathrm{B}$ promoter site governs the period of oscillations and is set to its known value $k_{\text {off }} \sim 0.1 \mathrm{~min}^{-1}$ generating 1-2-h-long oscillations consistent with single cell experiments [29-31]. The slowest time scale in the feedback loop corresponds to the degradation of $m$ RNA and the fastest time scales correspond to the various binding events of $\mathrm{NF} \kappa \mathrm{B}$ to $\mathrm{I} \kappa \mathrm{B}$ and to the DNA sites. In our calculations, dissociation from DNA bound sites can occur by passive unbinding with unimolecular kinetics
$D^{\text {on }} \stackrel{k_{\text {doff }}}{\longrightarrow} D^{\text {off }}+\mathrm{NF} \kappa \mathrm{B}$ or by molecular stripping, i.e., active $\mathrm{I} \kappa \mathrm{B}$ concentration dependent dissociation with bimolecular kinetics $\mathrm{I} \kappa \mathrm{B}+D^{\text {on } \stackrel{k_{s}}{\rightarrow}} D^{\text {off }}+\mathrm{I} \kappa \mathrm{B}-\mathrm{NF} \kappa \mathrm{B}$. The rate of $\mathrm{NF} \kappa \mathrm{B}$ removal is given by $k_{\mathrm{doff}}+k_{s} n_{I} n_{D}$, where $k_{s}=0$ corresponds to the situation with the molecular stripping absent and $k_{s} \neq 0$ when the molecular stripping is present (Fig. 1). The $n_{I}$ stands for number of $\mathrm{I} \kappa \mathrm{B}$ and $n_{D}$ for number of DNA-bound decoys. Using the equilibrium thermodynamic relationship $k_{\mathrm{don}} / k_{\mathrm{doff}}=e^{-\beta \Delta F}$ (which holds in the absence of molecular stripping under in vitro experimental conditions) one sees that molecular stripping can drastically alter the genomic regulatory landscape of $\mathrm{NF} \kappa \mathrm{B}$, making it kinetically controlled and dependent on the incoming flux of $\mathrm{I} \kappa \mathrm{B}\left(k_{s}\right)$ instead of the binding affinities $\Delta F$.

External stimulation of the network is modeled by setting the value of $\alpha$, the rate of degradation of $\mathrm{I} \kappa \mathrm{B}$ in the bound $\mathrm{NF} \kappa \mathrm{B}-\mathrm{I} \kappa \mathrm{B}$ complex. In real cells there are many sources of stimulation such as exposure to cytokines, free radicals, etc. All of these stimuli cause $\mathrm{I} \kappa \mathrm{B}$ to be marked for degradation, thus freeing the cytoplasmic $\mathrm{NF} \kappa \mathrm{B}$ which then diffuses into the nucleus to begin the regulatory cycle. Under steady stimulation (setting $\alpha=$ const) the network settles into a self-sustained oscillatory mode with periodic production and degradation of $\mathrm{I} \kappa \mathrm{B}$ molecules. The termination of stimulation corresponds to cessation of the degradation of $\mathrm{I} \kappa \mathrm{B}$ by setting $\alpha$ to zero. Figure 2(a) shows a sample of 50 trajectories of the time course of $\mathrm{NF} \kappa \mathrm{B}$-bound decoys. These trajectories are initiated from a state where all of the decoy sites are occupied by $\mathrm{NF}_{\kappa} \mathrm{B}$ with the remaining $\mathrm{NF} \kappa \mathrm{B}$ molecules being complexed with $\mathrm{I} \kappa \mathrm{B}$. We compare the situations with and without molecular stripping when the network is under constant external stimulation or transiently after that stimulation has been terminated. By looking at the switching after the stimuli have been terminated one immediately sees that even with our very conservative estimate of decoy residence times there is time-scale crisis for clearing out the decoys in the case of broadcasting network with no stripping. The time to turn off all the target genes exceeds by far the time scales of oscillation and gene expression $(\sim 120-150 \mathrm{~min})$. Meanwhile, when there is molecular stripping the switching off of all the decoy and target genes takes place in under $\sim 20 \mathrm{~min}$.

These time scales are very much in harmony with experiments of Dembinski et al. [34] and Fagerlund et al. [35]. These experiments carried out on single cells have probed the differences in nuclear clearance of $\mathrm{NF} \kappa \mathrm{B}$ for cells having wild type $\mathrm{I} \kappa \mathrm{B}$ versus a mutated [34] $\mathrm{I} \kappa \mathrm{B}$ or genetically engineered forms [35]. Both experiments show that cells with wild type $\mathrm{I} \kappa \mathrm{B}$ rapidly and robustly clear $\mathrm{NF} \kappa \mathrm{B}$ from the nucleus within a 20-30-min window while cells in which natural $\mathrm{I} \kappa \mathrm{B} \alpha$ has been replaced by $\mathrm{I} \kappa \mathrm{B} \beta$ which is lacking a PEST sequence (amino acid sequence rich in $\mathrm{P}, \mathrm{E}, \mathrm{S}, \mathrm{T}$ residues), which is crucial for molecular stripping [36], require more than $\sim 120-150 \mathrm{~min}$ in order to clear the $\mathrm{NF} \kappa \mathrm{B}$ from the nucleus.

The regime with high on rates [Fig. 2(b)] poses even more serious problems for general broadcasting networks because the rebinding events can make the clearance glassy (see next section), leading to clearance times which, vastly exceed the lifetime of cells. For the case of steady stimulation, when only passive release occurs, there are only highly stochastic oscillations with partial clearance of bound decoys. In contrast, 

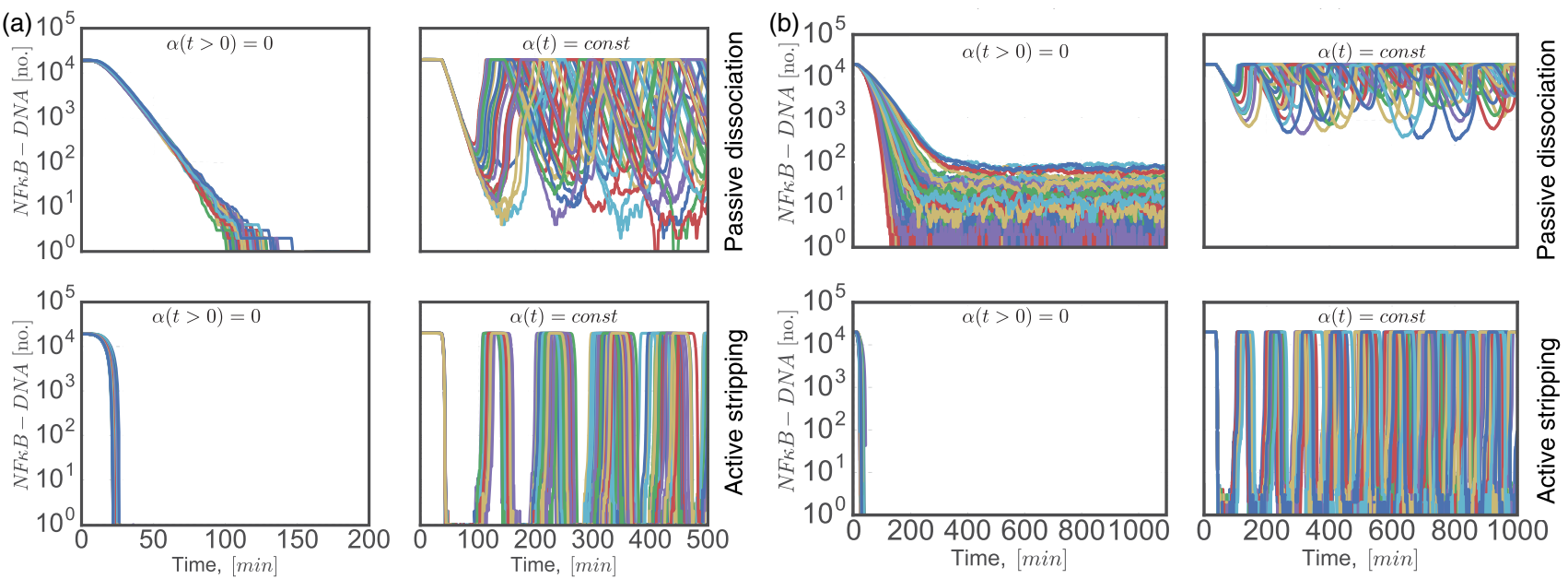

FIG. 2. Shown are 50 representative trajectories of NF $\kappa$ B-DNA complexes in broadcasting networks. We have examined cases of networks with passive dissociation (no stripping), $k_{s}=0 \mu \mathrm{M}^{-1} \min ^{-1}$ [first row, (a, b)], and with stripping, $k_{s}=10 \mu \mathrm{M}^{-1} \mathrm{~min}^{-1}$ [second row, (a, b)] under conditions of either terminated $(\alpha=0)$ or continuous $(\alpha>0)$ stimulation. (a) The slow on-off limit where rebinding of NF $\kappa$ B to DNA sites is negligible $\left(k_{\mathrm{doff}}=10 \mathrm{M}^{-1} \mathrm{~min}^{-1}\right)$ and the unbinding rates for all the bound decoys are $k_{\mathrm{doff}}=0.1 \mathrm{~min}^{-1}$. (b) The fast on-off rate limit where clearance times are dominated by frequent rebinding events $\left(k_{\mathrm{don}}=10^{3}-10^{4} \mathrm{M}^{-1} \mathrm{~min}^{-1}\right)$ of NF $\kappa \mathrm{B}$ to DNA sites and the unbinding rates for all the bound decoys are set to be $k_{\mathrm{doff}}=10 \mathrm{~min}^{-1}$.

when molecular stripping is included in the model, the network displays ultrasensitive and highly regular pulsatile behavior in which decoys and targets are fully cleared out in oscillations with a well defined period [Figs. 2(a) and 2(b)] which is again more consistent with the single cell experiments showing similarly small period variance for nuclear $\mathrm{NF} \kappa \mathrm{B}[29,30]$. The differences between passive release switches and switches that employ molecular stripping for decoy clearance are explored in the next section.

\section{ACTIVE REGULATION OF BOUND TRANSCRIPTION FACTORS RESOLVES THE TIME-SCALE CRISIS IN BROADCASTING NETWORKS}

As we saw in the previous section, without molecular stripping the very large number of binding sites and the consequent long time to clear them pose serious problems for reliable operation of eukaryotic broadcasting switches. To explore the time-scale crisis more comprehensively we vary the dissociation rates of bound decoys over a wider range $\left[10^{-2}-10\right]$ going from normal dissociation rates to very fast rates. To quantify the robustness of the switching process we compute the mean first passage times for clearing all the decoys of their bound $\mathrm{NF}_{\kappa} \mathrm{B}$ starting from a fully occupied decoy state once the stimulus has been terminated by setting $\alpha(t>0)=0$ in the model. We computed over $10^{4}$ trajectories for each setup and measured the mean and coefficient of variation of the clearance time for different unbinding rates going from the regime with no stripping $\left(k_{s}=0 \mathrm{M}^{-1} \mathrm{~min}^{-1}\right)$ to that with moderate molecular stripping $\left(k_{s}=10 \mathrm{M}^{-1} \mathrm{~min}^{-1}\right)$. Only for extremely fast intrinsic dissociation rates is the system able to function without molecular stripping (Fig. 3). For more realistic values of the dissociation rates from effective decoys ( 0.01-0.1) without stripping, a time-scale crisis arises in which some target genes take dramatically more time to clear than the basic gene expression time scale or the time scale of oscillations. Once stripping is allowed the switch's behavior is insensitive to orders of magnitude variation of molecular stripping rates [Fig. 3(a)]. Without stripping, the need for the multiplicity of binding sites to unbind stochastically makes turning off the targets corresponding to these sites highly unpredictable with some genes lingering in their active states for extremely long times. This lingering effect is quantified by the coefficient of variation of clearance times as a function of unbinding rates shown in Fig. 3(b). From Fig. 3 we see that the switch without molecular stripping gets noisier for sufficiently slow unbinding rates with the coefficient of variation diverging when the rates of $\mathrm{NF} \kappa \mathrm{B}$ dissociation from decoys become slow. This behavior implies that different cells in the population turn off their $\mathrm{NF} \kappa \mathrm{B}$ targets at random with some targets remaining on for much longer times than others do. Introducing molecular stripping, on the other hand, curbs the noise consistently throughout the range of unbinding rates.

The divergence of the coefficient of variation for the network with no stripping has two origins, the large number of decoys and their individually slow dissociation rates. To see how these two factors contribute to the mean and coefficient of variation, we simulate the network with no stripping while systematically varying the number of decoys and the dissociation rates (Fig. 4). Within the biologically relevant parameter range the effect of this divergence is apparent for decoy numbers in the range of $10^{3}-10^{4}$ and for dissociation rates from these decoys being $\sim 10^{-2} \mathrm{~min}^{-1}$ [Fig. 4(b)]. Surprisingly, when the dissociating rates are on the slow end of the spectrum, of the order of $\sim 10^{-2} \mathrm{~min}^{-1}$, the mean clearance time already exceeds hours even when there is a moderately low number of decoys $10^{2}-10^{3}$ [Fig. 4(a)].

The stochastic nature of clearance is evident when we examine the stochastic dynamics of the occupancies of each individual decoy site [Fig. 5(a)]. Looking at the time trajectory of the occupancy of individual sites (quantified via probabilities of survival) reveals that some sites can remain occupied on time 
(a)

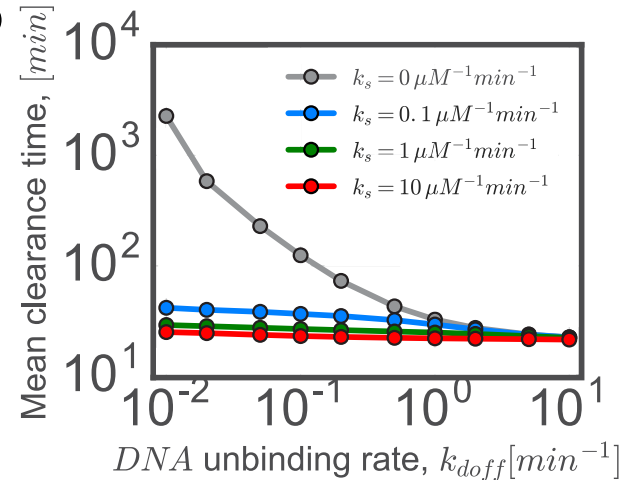

(b)

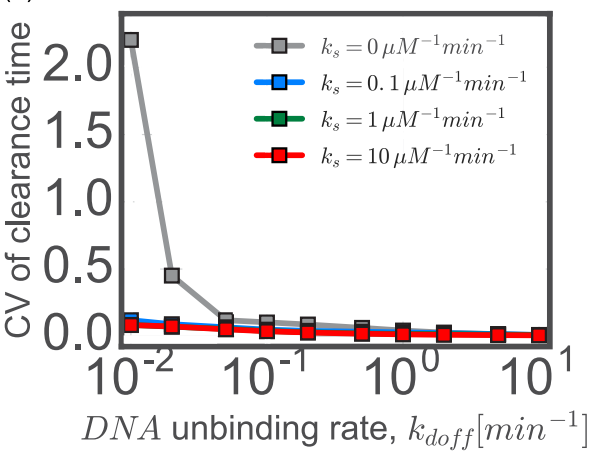

(c)

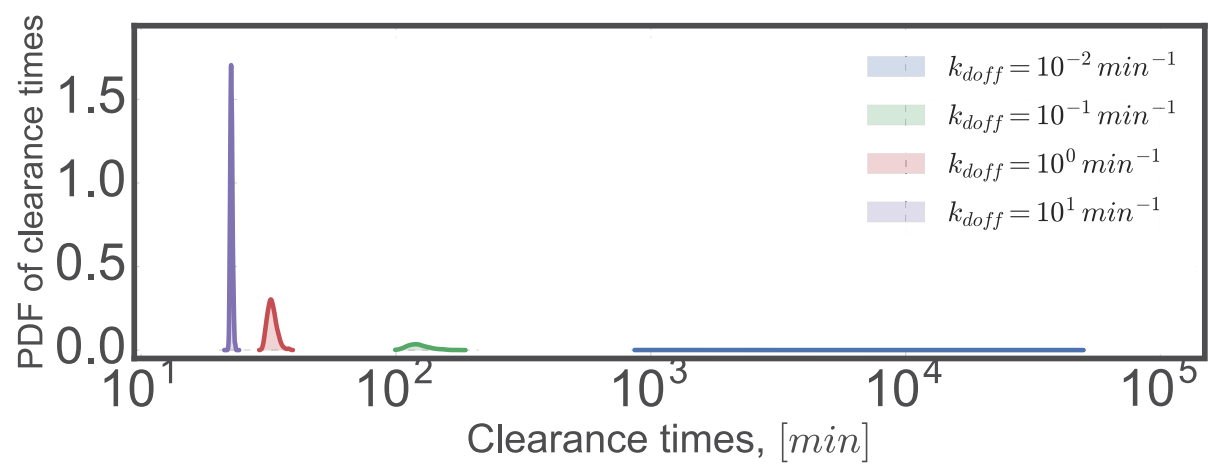

FIG. 3. (a) The dependence of the mean clearance time of occupied decoys NF $\kappa$ B-DNA on the unbinding rate for cases of no stripping and stripping with rates $k_{s}$. (b) The dependence of the standard deviation of clearance times for cases of no stripping and stripping with rates $k_{s}$. (c) The probability distributions corresponding to different unbinding rates in the case with no stripping, $k_{s}=0$.

scales comparable to complete clearance without ever having undergone unbinding or rebinding [37]. These "stragglers" would potentially pose problems for broadcasting networks without molecular stripping. When there is molecular stripping in the model, as we have seen from Fig. 3, clearance happens rapidly and reliably. These features are reflected in the rapidly decaying survival probabilities for individual DNA binding sites [Fig. 5(b)]. While the stragglers contribute greatly to
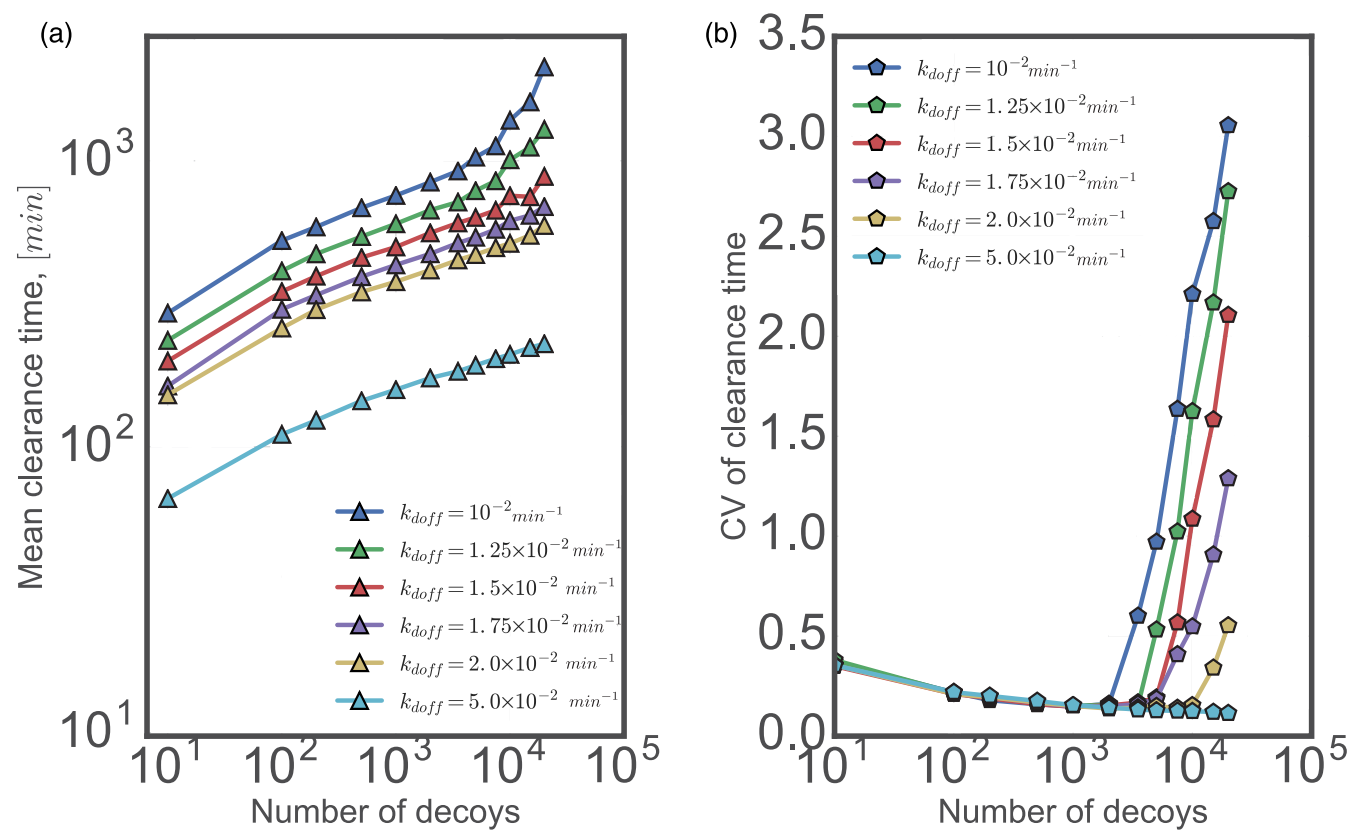

FIG. 4. (a) Mean and (b) coefficient of variation for the broadcasting network with no stripping $\left(k_{s}=0 \mu \mathrm{M}^{-1} \mathrm{~min}^{-1}\right)$ as a function of numbers of decoys. 
(a)

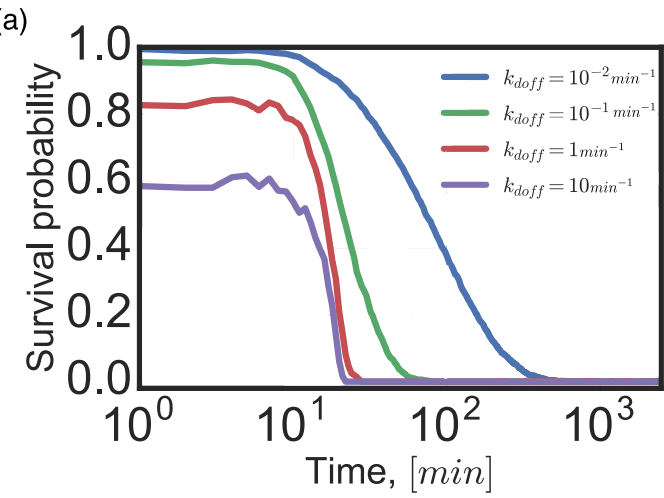

(c)

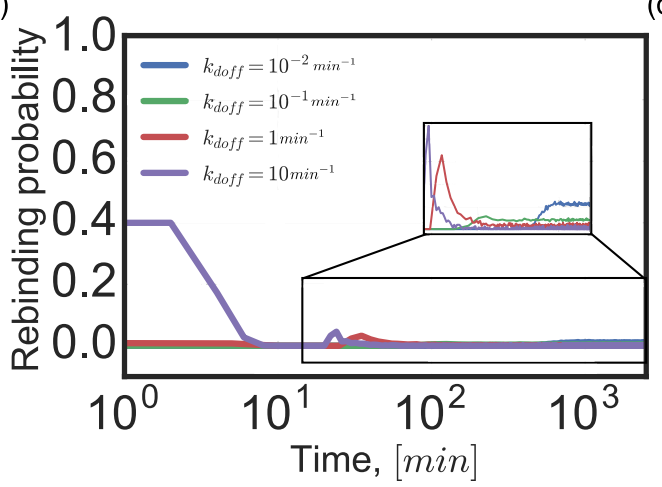

(b)

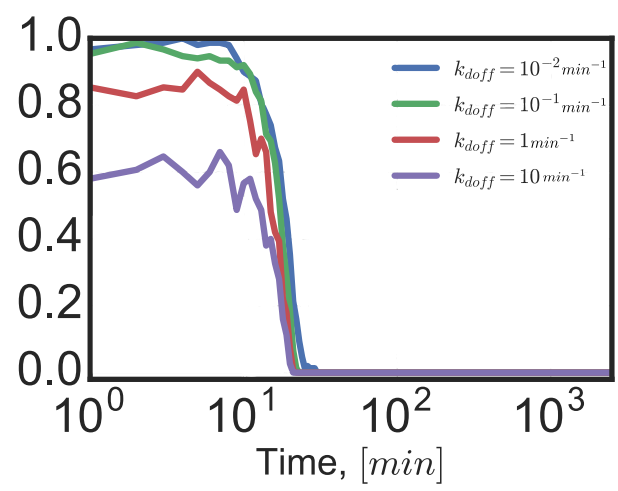

(d)

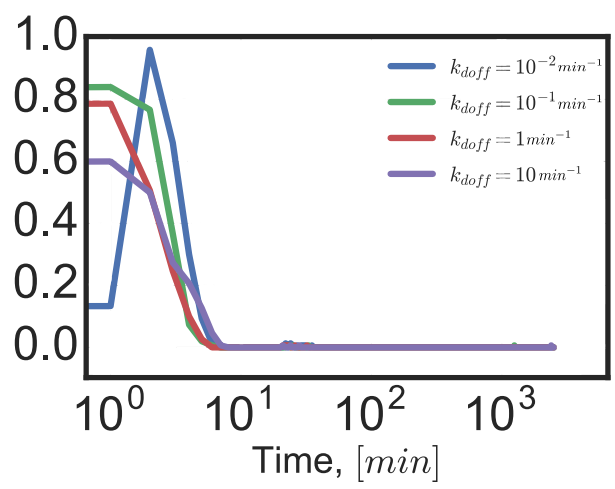

FIG. 5. The survival probabilities of single bound decoy sites as a function of time for the networks (a) with no stripping and (b) with stripping. Rebinding probabilities are computed as a function of time for the cases (c) with no stripping and (d) with stripping. Different curves correspond to different dissociation rates of the $\mathrm{NF} \kappa \mathrm{B}$ bound decoys.

the mean clearance time in the case of a broadcasting network with no stripping [37], they are not the only source of variance. Rebinding events, where free $\mathrm{NF} \kappa \mathrm{B}$ binds back to the newly cleared sites, not only slow down the complete clearance of bound $\mathrm{NF} \kappa \mathrm{B}$ but also make the clearance times highly unpredictable, which is reflected in the divergence of the coefficient of variation seen in Fig. 4. These rebinding events are purely stochastic in their origin, happening mostly at the later stages of clearance [Fig. 5(c)] when the large number of unoccupied decoy sites start to compete with the $\mathrm{I} \kappa \mathrm{B}$ for the free $\mathrm{NF} \kappa \mathrm{B}$ [37].

The rebinding induced time-scale crisis for clearance becomes severe in the limit when the binding on rates are much larger than the rate of $\mathrm{I} \kappa \mathrm{B}$ binding to $\mathrm{NF} \kappa \mathrm{B}\left(k_{\mathrm{don}} \gg k_{f n}\right)$ [Fig. 2(b)]. In this hyper-fast binding regime the clearance becomes much more sluggish and largely insensitive to the off rates $k_{\text {doff }}$ because of the very high rebinding probabilities [37]. In this limit terminating the stimulus stochastically settles the network into various metastable steady states with significant numbers of long lived nuclear $\mathrm{NF} \kappa \mathrm{B}$ [Fig. 2(b)]. The kinetic experiments of Bosisio et al. [38] conducted on living cells have shown that such a hyperdynamic scenario could be a possibility where the residence time of $\mathrm{NF} \kappa \mathrm{B}$ molecules is largely maintained by the high on rates while at the same time the dissociation is seen to happen at a faster time scale that would be inferred from in vitro measurements. There can be additional biological complexities behind the large residence times of transcription factors [7,39] such as presence of affinity enhancing cofactors, sliding, intersegmental transfer, and chromatin dynamics just to name a few. Regardless of the detailed molecular mechanism of transcription factor DNA interaction, however, we see that in the absence of stripping whenever there is a large number of decoys there will be a large residence time, therefore posing a fundamental challenge for the full clearance of transcription factors. When there is molecular stripping, the rebinding events simply do not matter very much because the rate of clearance is too rapid for rebinding to influence the clearance process [Fig. 5(d)].

Now we turn to investigating how molecular stripping changes the stochastic behavior of the switch when the broadcasting system displays self-sustained pulsatile behavior [31]. Keeping the degradation rate $\alpha(t>0)=0.25 \mathrm{~min}^{-1}$ constant in the simulation leads to oscillations of the nuclear $\mathrm{NF} \kappa \mathrm{B}$ with a period of $\sim 2 \mathrm{~h}$ [37] consistent with the commonly adopted experimental setup $[29,30]$. In the present paper, however, we are interested in the consequences for the residence times of $\mathrm{NF} \kappa \mathrm{B}$ at genomic binding sites. Therefore we also examine the oscillations of the occupation of decoy states as a function of unbinding rates. The mean fraction of the time that the sites are completely cleared [Fig. 6(a)] provides a good way to quantify how long the genes will be transcriptionally silent on average under the external stimulation. Different patterns of pulses of $\mathrm{NF} \kappa \mathrm{B}$ are thought to activate different patterns of genes for downstream signaling [31]. Experiments suggest that the temporal dynamics of $\mathrm{NF} \kappa \mathrm{B}$ has information that can be utilized for decision making by the cell. For the broadcasting network without any molecular stripping the mean fraction of completely cleared times is essentially zero over a broad 

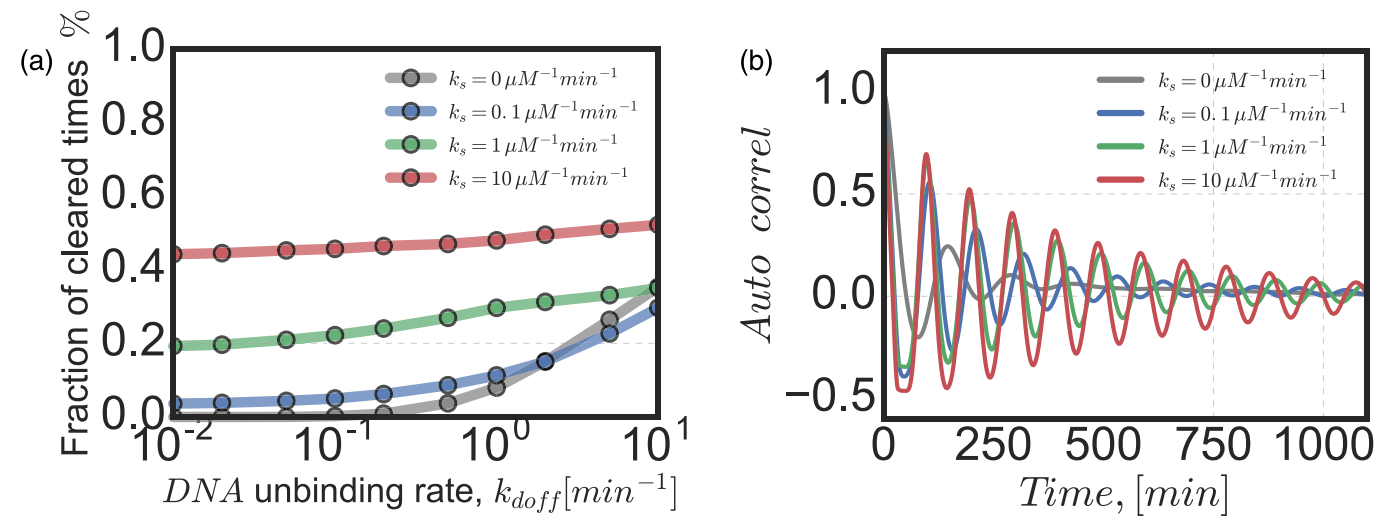

FIG. 6. (a) Dependence of the mean fraction of cleared times on the decoy unbinding rate computed for networks' different stripping rates $k_{s}$. (b) Temporal autocorrelation function of DNA bound NF $\kappa \mathrm{B}$ molecules for the unbinding rate of $k_{\text {doff }}=0.1 \mathrm{~min}^{-1}$.

range of dissociation rates as there are always some $\mathrm{NF} \kappa \mathrm{B}$ molecules bound to the decoy sites at all times. Only in the fast dissociation limit do the oscillations become clearly pulsatile, alternating between completely cleared and occupied states. With molecular stripping, due to the fast turnover of the NF $\kappa \mathrm{B}$ the oscillations become ultrasensitive over a wide range of values of molecular stripping rate.

A curious situation occurs in the fast dissociation limit where the full genomic clearance of the network with slow molecular stripping becomes comparable to or slightly lower than the network without stripping. This phenomenon occurs because the stripping of the promoter site for $\mathrm{I} \kappa \mathrm{B}$ reduces the overall rate of stripping of the remaining decoy sites beyond that of passive dissociation. We verified that this is appropriate explanation by simulating what happens when one selectively turns off the stripping only at the promoter site. This change makes the mean wait times for all $k_{s}>0$ even longer [37]. One may imagine similar strategies used by the cell where the signals are being broadcast to many targets with a few selected ones gaining special protection by modification of chromatin structure.

The autocorrelation of the pulses is another quantity that shows how rapidly noise randomizes the relative phases of different oscillators [Fig. 6(c)]. Again, networks with molecular stripping hold the phases correlated over much longer times than happens in the absence of stripping where it takes at most two to three pulses for phases to become completely uncorrelated.

\section{THE NONEQUILIBRIUM NATURE OF SWITCHES IN A BROADCASTING SYSTEM}

Molecular stripping modifies the often adopted equilibrium picture of gene switches by adding to the otherwise reversible step of transcription factor binding or unbinding a microscopically irreversible step. One should note, however, that in reality molecular stripping just like any other chemical reaction must have a finite backward rate, which in the case of stripping and a few other reactions in our network is thought to be vanishingly small. The network as a whole is driven outside of equilibrium by continuous stimulation from the outside. Therefore there is no question that both with and without stripping the $\mathrm{NF} \kappa \mathrm{B}$ regulatory network operates under highly nonequilibrium con- ditions where molecules are constantly being pumped into and degraded out of the system, thereby guaranteeing the stability of steady oscillatory or homeostatic states. Quantifying the extent of irreversibility of genetic networks is not trivial partly because of the great complexity of real biological networks with many steps that individually dissipate energy that could potentially contribute to the feedback cycle.

Since the thermodynamic affinities of DNA sites are commonly used for thinking about gene regulation, we would like to quantify how informative such quantities are under these more general nonequilibrium conditions.

When there is no stripping and when binding and spontaneous unbinding are fast, the equilibrium affinities are strongly correlated with the mean clearance time, with higher affinities corresponding to slower clearance and vice versa [Fig. 7(a)]. On the other hand, when binding and spontaneous unbinding are slow, the mean clearance times only weakly depend on the equilibrium affinities but are instead dictated by the dissociation rates. In a sense when there is molecular stripping the concept of affinity loses its meaning altogether. Both the means and the variances of the clearance times become independent of the equilibrium affinities [Fig. 7(b)]. As discussed in the previous section the hyperdynamic binding with high on rates can dramatically slow down the clearance as is seen by the plateauing of the clearance times at fast off rates [Fig. 7(a)]. This slowdown is caused by frequent DNA rebinding reactions which make overall clearance a rare event [37]. At the steady state, stripping causes the mass action ratios to be governed only by the binding on rates since the effective off rates from all the decoys become equal [Figs. 7(c) and 7(d)]. These rates are very often diffusion limited. The equilibrium dissociation constant would clearly not be a very reliable measure of the "strength" of binding for these dynamical situations.

\section{CONCLUSION}

In this paper we have studied the stochastic dynamics of the broadcasting genetic network centered on transcription factor $\mathrm{NF} \kappa \mathrm{B}$. Latest genomic studies have revealed that $\mathrm{NF} \kappa \mathrm{B}$ is a master regulator which broadcasts information to its numerous targets via binding to a huge number of DNA 
(a)

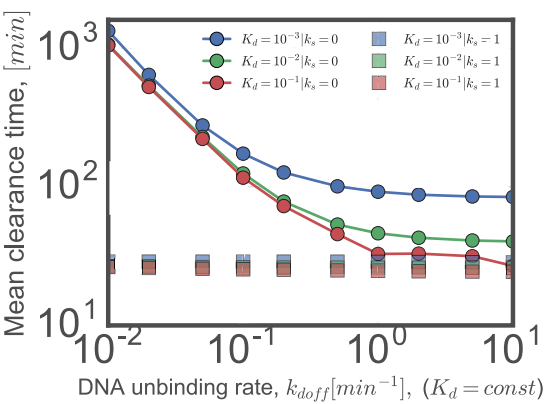

(c)

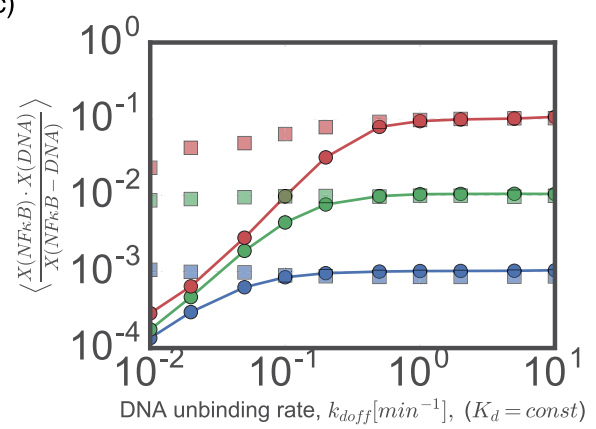

(b)

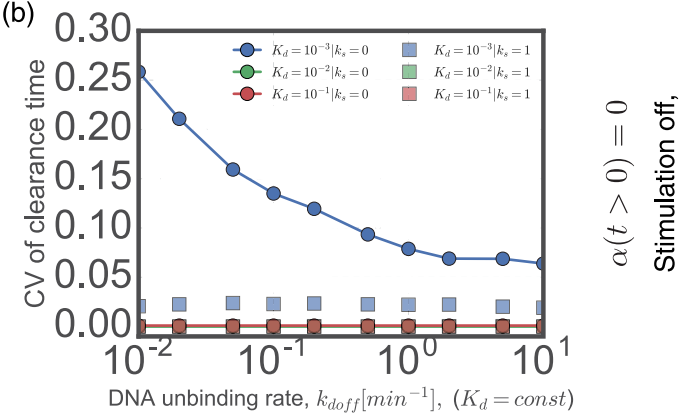

(d)

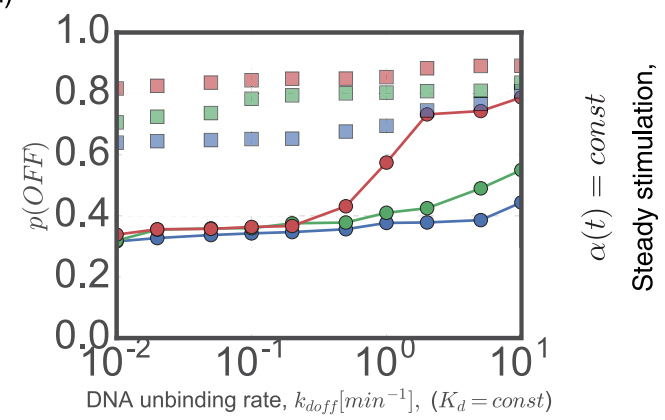

FIG. 7. The values of the (a) mean clearance time and (b) coefficient of variation of the network with terminated stimuli as a function of dissociating rates $k_{\text {doff }}$ with fixed thermodynamic affinities $K_{d}=k_{\text {doff }} / k_{\text {don }}=$ const. (c) Mass action ratio of decoy-NF $\kappa$ B quasiequilibrium, with the corresponding macroscopic dissociation as a function of dissociation rates $k_{\text {doff }}$. (d) Probability of the unoccupied $I \kappa \mathrm{B}$ promoter.

sites having coding, downstream regulatory consequences, and nonfunctional effects. Single cell experiments have also shown that under steady stimulation the network exhibits self-sustained oscillations but that once the external stimuli are terminated the network rapidly switches into a nonoscillatory homeostatic state.

In this paper we have shown that passive dissociation of transcription factors can lead to a time-scale crisis for broadcasting signals to targets if the targets and decoys are too numerous. We show the generality of the time-scale crisis which within the thermodynamic paradigm of gene regulation is solved only in the limit of extremely fast dissociation and absence of any rebinding events to DNA sites. We thus argue for the case of nonequilibrium kinetic control as a means of regulating large genomic sites in networks that broadcast signals to a large number of sites. In the case of the $\mathrm{NF} \kappa \mathrm{B}$ network, molecular stripping by $\mathrm{I} \kappa \mathrm{B}$ solves the time-scale crisis for clearance and leads to fast responses and ultrasensitive oscillations with well defined periods. Our results are in harmony with recent in vivo single cell experiments where the stripping process has been perturbed specifically $[34,35]$. Finally, we predict that networks for which molecular stripping or similar mechanisms for assisted dissociation will be discovered will likewise show robustness at transitioning between steady states and oscillatory states with little to no dependence on the distribution of binding free energies and the number of broadcasting regulatory sites.

\section{ACKNOWLEDGMENTS}

We thank Elizabeth Komives for many insightful discussions about the biochemistry of the $\mathrm{NF} \kappa \mathrm{B} / \mathrm{I} \kappa \mathrm{B}$ broadcasting network. We also acknowledge financial support by the D. R. Bullard-Welch Chair at Rice University Grant No. C-0016 and PPG Grant No. P01 GM071862 from the National Institute of General Medical Sciences.
[1] M. Ptashne, A Genetic Switch: Phage Lambda Revisited (Cold Spring Harbor Laboratory, Cold Spring Harbor, New York, 2004), Vol. 3.

[2] G. K. Ackers, A. D. Johnson, and M. A. Shea, Proc. Natl. Acad. Sci. USA 79, 1129 (1982).

[3] L. Bintu, N. E. Buchler, H. G. Garcia, U. Gerland, T. Hwa, J. Kondev, and R. Phillips, Curr. Opin. Genet. Dev. 15, 116 (2005).

[4] W. Gilbert and B. Müller-Hill, Proc. Natl. Acad. Sci. USA 56, 1891 (1966).

[5] F. Jacob and J. Monod, J. Mol. Biol. 3, 318 (1961).

[6] G. D. Stormo and Y. Zhao, Nat. Rev. Gen. 11, 751 (2010).
[7] A. Coulon, C. C. Chow, R. H. Singer, and D. R. Larson, Nat. Rev. Gen. 14, 572 (2013).

[8] T. L. Lenstra, J. Rodriguez, H. Chen, and D. R. Larson, Ann. Rev. Biophys. 45, 25 (2016).

[9] K. M. Lelli, M. Slattery, and R. S. Mann, Annu. Rev. Gen. 46, 43 (2012).

[10] T. Ha, Cell 154, 723 (2013).

[11] P. Hammar, M. Walldén, D. Fange, F. Persson, Ö. Baltekin, G. Ullman, P. Leroy, and J. Elf, Nat. Genet. 46, 405 (2014).

[12] T.-Y. Chen, A. G. Santiago, W. Jung, Ł. Krzemiński, F. Yang, D. J. Martell, J. D. Helmann, and P. Chen, Nat. Commun. 6, 7445 (2015). 
[13] J. Estrada, F. Wong, A. DePace, and J. Gunawardena, Cell 166, 234 (2016).

[14] S. A. Cepeda-Humerez, G. Rieckh, and G. Tkačik, Phys. Rev. Lett. 115, 248101 (2015).

[15] V. Alverdi, B. Hetrick, S. Joseph, and E. A. Komives, Proc. Natl. Acad. Sci. USA 111, 225 (2014).

[16] D. A. Potoyan, W. Zheng, E. A. Komives, and P. G. Wolynes, Proc. Natl. Acad. Sci. USA 113, 110 (2016).

[17] S. Bergqvist, V. Alverdi, B. Mengel, A. Hoffmann, G. Ghosh, and E. A. Komives, Proc. Natl. Acad. Sci. USA 106, 19328 (2009).

[18] J. S. Graham, R. C. Johnson, and J. F. Marko, Nucleic Acids Res. 39, 2249 (2011).

[19] M. J. McCauley, E. M. Rueter, I. Rouzina, L. J. Maher, and M. C. Williams, Nucleic Acids Res. 41, 167 (2013).

[20] C. P. Joshi, D. Panda, D. J. Martell, N. M. Andoy, T.-Y. Chen, A. Gaballa, J. D. Helmann, and P. Chen, Proc. Natl. Acad. Sci. USA 109, 15121 (2012).

[21] J. J. Loparo, A. W. Kulczyk, C. C. Richardson, and A. M. van Oijen, Proc. Natl. Acad. Sci. USA 108, 3584 (2011).

[22] D. D. MacDougall and R. L. Gonzalez, J. Mol. Biol. 427, 1801 (2015).

[23] H. L. Pahl, Oncogene 18, 6853 (1999).

[24] R. Martone, G. Euskirchen, P. Bertone, S. Hartman, T. E. Royce, N. M. Luscombe, J. L. Rinn, F. K. Nelson, P. Miller, M. Gerstein, S. Weissman, and M. Snyder, Proc. Natl. Acad. Sci. USA 100, 12247 (2003).

[25] T. Siggers, A. B. Chang, A. Teixeira, D. Wong, K. J. Williams, B. Ahmed, J. Ragoussis, I. A. Udalova, S. T. Smale, and M. L. Bulyk, Nat. Immun. 13, 95 (2012).

[26] B. Zhao, L. A. Barrera, I. Ersing, B. Willox, S. C. Schmidt, H.
Greenfeld, H. Zhou, S. B. Mollo, T. T. Shi, K. Takasaki et al., Cell Rep. 8, 1595 (2014).

[27] A. Antonaki, C. Demetriades, A. Polyzos, A. Banos, G. Vatsellas, M. D. Lavigne, E. Apostolou, E. Mantouvalou, D. Papadopoulou, G. Mosialos et al., J. Biol. Chem. 286, 38768 (2011).

[28] S. C. Gupta, C. Sundaram, S. Reuter, and B. B. Aggarwal, Biochim. Biophys. Acta 1799, 775 (2010).

[29] D. Nelson, A. Ihekwaba, M. Elliott, J. Johnson, C. Gibney, B. Foreman, G. Nelson, V. See, C. Horton, and D. Spiller, Science 306, 704 (2004).

[30] R. A. Kellogg and S. Tay, Cell 160, 381 (2015).

[31] S. Zambrano, I. De Toma, A. Piffer, M. E. Bianchi, and A. Agresti, Elife 5, e09100 (2016).

[32] A. Hoffmann, A. Levchenko, M. L. Scott, and D. Baltimore, Science 298, 1241 (2002).

[33] D. T. Gillespie, J. Phys. Chem. 81, 2340 (1977).

[34] H. E. Dembinski, K. Wismer, J. D. Vargas, G. W. Suryawanshi, N. Kern, G. Kroon, H. J. Dyson, A. Hoffmann, and E. A. Komives, Proc. Natl. Acad. Sci. USA 114, 1916 (2017).

[35] R. Fagerlund, M. Behar, K. T. Fortmann, Y. E. Lin, J. D. Vargas, and A. Hoffmann, J. Roy. Soc. Int. 12, 20150262 (2015).

[36] D. A. Potoyan, W. Zheng, D. U. Ferreiro, P. G. Wolynes, and E. A. Komives, J. Phys. Chem. B 120, 8532 (2016).

[37] See Supplemental Material at http://link.aps.org/supplemental/ 10.1103/PhysRevE.96.052305 for figures and additional information

[38] D. Bosisio, I. Marazzi, A. Agresti, N. Shimizu, M. E. Bianchi, and G. Natoli, EMBO J. 25, 798 (2006).

[39] F. Mueller, T. J. Stasevich, D. Mazza, and J. G. McNally, Crit. Rev. Biochem. Mol. Biol. 48, 492 (2013). 